Manual of Histological Techniques. JD Bancroft and HC Cook. (Pp 274; £12.50.) Churchill Livingstone. 1984 .

This is restricted to histological staining methods, mainly dye staining techniques with some enzyme histochemistry and a very little immunocytochemistry. It has a similar format to its more useful companion volume "Theory and Practice of Histological Techniques" by Bancroft and Stevens but is cheaper, smaller, and soft back; it contains much the same staining methods but lacks tissue processing and sectioning as well as all the other wider aspects of histopathological technique. Little has been gained and much has been lost in this return to traditional dye staining methods. This book does not reflect the great advances that have been made in histopathology and trainees should be encouraged to read more widely.

\section{RAB DRURY}

Molecular Basis of Lysosomal Storage Disorders. Ed JA Barranger and RO Brady. (Pp 502; £31·50.) Academic Press Inc. 1984.

This volume is the proceedings of a conference on lysosomal storage diseases which was held at the National Institutes of Health, Bethesda, in September 1983 and in which many of the leading workers in the field took part. It is an excellent summary of the position as it was at that date and although one regrets the time lapse in its appearance, it is still a valuable and topical publication for those interested in lysosomal enzymes and the inborn errors of metabolism which are due to metabolic lesions involving them. Although the first of the activator proteins which are necessary for the activity of lysosomal enzymes was isolated as long as 20 years ago, this subject has advanced rapidly in recent years and there are outstanding contributions on it in this volume. The mechanisms which route lysosomal enzyme proteins to the prelysosomal compartment and away from the pathways followed by secretory glycoproteins are a field in which there was much to report and which offer a basis for a whole new range of inborn errors of metabolism of which I-cell disease (mucolipidosis II) can be considered to be the archetype.

The four chapters on the biosynthesis of lysosomal enzymes with particular reference to hexosaminidase and $\alpha$-glucosidase are particularly welcome.

The section on molecular genetic aspects is important for clinical molecular geneticists because of the valuable accounts of the progress with respect to the cloning of genes for the enzymes, lesions of which are responsible for lysosomal storage diseases. The chapter on strategies for the molecular cloning of low abundance messenger RNAs is particularly important.

The section on treatment deals with bone marrow transplantation in the treatment of lysosomal storage diseases as exemplified by the continuing work on Hurler disease and a report of the use of this approach in a case of Gaucher disease. There is also an account of the present position with respect to enzyme supplementation in Gaucher disease. The position with respect to these subjects is slowly being clarified.

This book is firmly recommended for clinical and laboratory investigators working on the lysosomal storage diseases and the more applied aspects of lysosomal physiology. Other readers, particularly paediatricians and biochemists, will also find much of interest.

RWE WATTS

Hematopoiesis. Methods in Hematology. Ed David W Golde. (Pp 361; £38.) Churchill Livingstone. 1984.

This valuable addition to the "Methods in Hematology" series provides a comprehensive resumé of the methods used for the study of in vivo and in vitro haematopoiesis. One of the problems experienced by new workers in this field is that superficially minor technical aspects crucial to the success of a method are not fully discussed in original publications. This volume will do much to help in this regard, including as it does lists of possible faults, clear instructions for colony recognition, and advice on the preparation of the most mundane ingredients. There is also a short section on the methods for in vitro enrichment of human stem cells, although I suspect this is the one part of the book that will soon be out of date.

This book will not be a substitute for gaining experience in the field but will make the setting up of new methods much easier. Newcomers will find those chapters relevant to their interests essential reading, and the volume will be needed by most laboratories which grow blood cells.

IM FRANKLIN

The Physiology and Pathophysiology of the Skin. Vol 8. The Photobiology of the Skin Lasers and the Skin. Ed A Jarrett. (Pp 2704; £49.) Academic Press. 1984.
This book is the latest in a series which in the previous seven volumes has covered anatomically defined subjects (the epidermis, nerves, and blood vessels, the dermis and dendrocytes, sweat glands, the mucus membranes), and pathophysiological topics (skin permeation, transepidermal water loss, hormonal control of pigmentation, and the biology of melanoma). This volume covers photobiology (187 pages), and lasers (18 pages). The relative lengths of these contributions probably reflect the degree of current use of these two treatment modalities by clinical dermatologists.

The section on the photobiology of the skin is written by Brian Johnson, Senior Lecturer in Photobiology in the Department of Dermatology at the University of Dundee. It is currently commonplace for able and enthusiastic younger workers with medical qualifications to take a "sideways step", spending a year or two in a laboratory learning the scientific techniques of the new biology-gene cloning, monoclonal antibody production, oncogene detection, and the like. It is not common, however, for a scientist to take the time and trouble to become really well versed in the clinical problems related to his research area to such an extent that his knowledge is in many cases greater than that of medical colleagues. Dr Johnson has done just this and his approach is a welcome breath of fresh and authoritative air in the dermatological community. His contribution begins with essential background information on natural and artificial radiation, and moves to consider the effect of solar radiation on normal skin, and to the photosensitive disorders and the mechanisms thought to underly them. The important area of photosensitive drug reactions is covered particularly well and an excellent and comprehensive account of general phototherapy and photochemotherapy concludes his contribution.

The section on photosensitive drug reactions and their mechanisms could be read with advantage by all those involved in the prescription of the many potential photosensitisers, and the section on the value and side effects of photochemotherapy should be required reading for all those who currently operate 'PUVA' services. The section devoted to histological changes seen in UV exposed normal skin will obviously be of value and interest to diagnostic pathologists.

The section on lasers is also well handled by Dr John Cotterill, who is a Consultant Dermatologist at Leeds General Infirmary. It is divided mainly into two sections, one 
on the physics of laser production, and one on the types of laser currently available. Time will tell just how useful this treatment modality will be in the management of a variety of benign and malignant skin conditions.

A particularly attractive feature of the layout in this series is the placing of the references at the foot of the page carrying the relevant statement. This allows generous referencing without the tedious thumbing to the end of each chapter as one reads. The book is recommended. By current publishing standards it is reasonable value for money.

RONA M MACKIE

Clinical Biochemistry. Contemporary Theories and Techniques. Vol 3. Ed Herbert E Spiegel. (Pp 271; \$42.00.) Academic Press. 1984.

This book touches on the laboratory specialties of clinical biochemistry, micribiology, haematology, and immunology. Its broad coverage coupled with limitations of space mean that many of the descriptions are superficial. It is difficult to know for whom it is intended.

Two chapters are particularly concerned with clinical biochemistry; biochemical monitoring of cancer, and instrumentation in clinical chemistry. The former provides a clear, reasonably comprehensive and balanced coverage of a frequently reviewed subject. The latter review attempted an impossible task within the space available, and is in any case already three years out of date.

The interests of microbiologists account for chapters on chemical and physiochemical approaches to detecting and identifying causes of infection, and on serologic methods of diagnosis. However, modern biochemical techniques still only have a supportive role in microbiology. Both chapters would have benefited from greater use of diagrams, and more attention should have been paid to costs, especially of kit methods.

Haemoglobin structure and the haemoglobinopathies make up another chapter, and there is a distinctly selective review of monoclonal antibodies in clinical investigations. Both these subjects have been extensively reviewed elsewhere, and the monoclonal antibody field is moving fast.

All in all, I regret to say that this expensive book is of questionable value for money.

LG WHITBY
Viruses and Demyelinating Diseases. Ed CA Mims, ML Cuzner, and RE Kelly. (Pp 201; £22·50.) Academic Press. 1984.

This multiple author book is based on a symposium organised by the Multiple Sclerosis Society of Great Britain and Northern Ireland in Edinburgh, September 1982.

For many years viruses have been invoked in the aetiology of multiple sclerosis. The principal aim of these proceedings was therefore to review the current situation by considering, amongst other things, animal models of demyelination, antibody induced modulation of virus antigens, and defective replication and persistence of viruses in the central nervous system. There is also a very interesting review of the effect of chemical injury on oligodendrocytes.

Even though no virus or virus like agents or aetiologically significant antiviral antibodies have been identified, it is still thought that the most unifying hypothesis about multiple sclerosis has to do with virus infections in the central nervous system that trigger demyelination either as a 'bystander' effect or as a specific immune response.

This book gives a clear and up to date account of a complex subject and raises the distinct hope that a clear understanding of the pathogenesis of multiple sclerosis will become available in the not too distant future. It is therefore strongly recommended to neurologists, pathologists with an interest in the nervous system, and all neuroscientists.

DI GRAHAM

The Biochemical Effects of Drugs in Pregnancy. Vol 2. A Onnis and P Grella. (Pp 493; £45.) John Wiley. 1984.

The same team have produced a Volume 1 covering drugs acting on the nervous, cardiovascular, and haematopoietic systems. These books have been designed as reference books on the use of therapeutic drugs in pregnancy for "clinicians" which should include those in laboratory medicine. As a reference book it is well compiled. Each entry starts with a single sentence summary of recommendations. Thereafter possible teratogenic effects, pharmacological actions, and effects on human pregnancy and lactation are presented. A summary of animal pharmacology during pregnancy is included. It is emphasised that the information is not absolute and the results of animal experimentation cannot be directly applied to man, although such results remain our best guide.

The short preface, introduction, ant directions for use are good. The individua: entries are, on random sampling, usefü and well indexed. The bibliography cove the Italian and French as well as the Nort ern European and English language liter ture. It should be remembered that thes book is primarily a catalogue and not series of critical reviews. It is also, as admi乡? ted, not a comprehensive catalogue.

In laboratory medicine practice, the coincidence of a feto maternal abnormalitus and drug administration is a recurre problem. For such problems this book aff presumably Volume 1 are a useful source of information. The book(s) should ther fore be available in libraries directly of indirectly serving those working on obs tetric and paediatric problems.

Perhaps the last words should be reassuo ing and can be left to the authors; "In fact there are very few drugs which are able to produce malformations in human preso nancy".

RA HARKNE $\overrightarrow{8 \Omega}$

@

Special Tumors of the Head and Neck. PrQ ceedings of the 48th Annual Anatomic Pathology Slide Seminar of the America्m Society of Clinical Pathologists. John \& Batsakis and Vincent J Hyams. (Pp 11을 paperback $\$ 34 \cdot 00$.) Raven Press. 1984. 응

This book consists of a discussion of cases from a slide seminar presented at $ב$ meeting in 1982. Most of the lesions a rare. Some useful diagnostic problems are reviewed such as those posed by na:्लुl polyps with stromal atypia midfacial neirotising lesions, and fibromyxomato lesions of the sino nasal tract. The histolog ical appearances of the cases in the ligt microscope are, for the most part, adequately depicted. The use of additional diag nostic procedures receives surprisingly lif tle attention. The role of the electron mir: roscope is discussed in some cases, but $\overline{\text { nit }}$ receives little emphasis in others-notabPy olfactory neuroblastoma. Only one eled tron microscope photograph is included in the text. Immunohistochemical techniques attract limited comment even though they can be useful in at least 5 of the 25 cases that are discussed-extramedullary plas macytoma and extranodal lymphomas, metastatic carcinoma of the nasopharyn embryonal rhabdomyosarcoma, aß synoviosarcoma. Publication appears have been a slow process, and no new references have been added since 1982 . 응 RL CARTER 